\title{
Micromecanizado mediante láseres de femtosegundos de cerámicas LTCC. Fabricación de microcalentadores para un sensor de monóxido de carbono
}

\author{
DAVID GÓMEZ'1 IGOR GOENAGA', PER JOHANDER², CARMEN MOLDOVAN³ \\ 'Dpto. de Micro y Nanotecnologías, Fundación Tekniker, Av. Otaola 20, 20600 Eibar, Guipúzcoa (España) \\ 2 IVF - Industrial Research and Development Corporation, Argongatan 30, S43153 Molndal (Suecia) \\ ${ }^{3}$ National Institute for R\&D in Microtechnologies, Erou lancu Nicolae 32B, 077190 Bucarest (Rumanía)
}

\begin{abstract}
El presente trabajo pretende mostrar la procesabilidad de cerámicas de baja temperatura de sinterizado, LTCC (Low Temperature Co-fired Ceramics) mediante ablación láser con pulsos ultracortos (femtosegundos). Este tipo de material, de indudable interés tecnológico en el desarrollo de microsensores, presenta una alta fragilidad en el estado sinterizado, por lo que la utilización de métodos de contacto (fresado, torneado, etc.) para su mecanizado conduce a la fractura o la aparición de microgrietas y desconchamientos. El trabajo ha sido dividido en dos partes bien diferenciadas. Primeramente, se ha realizado un estudio del proceso, obteniéndose los parámetros fundamentales (el umbral de ablación y el coeficiente de incubación) y se ha procedido a una parametrización de la anchura y altura de microcanales en función de la densidad de energía y el avance relativo en el movimiento muestra-láser. Posteriormente, estos resultados han permitido el micromecanizado de estructuras sencillas, como cajeras, orificios y estructuras en "V". Finalmente, se presenta una aplicación práctica que muestra el interés tecnológico del proceso. Así, se han realizado microcalentadores en LTCC como etapa inicial en el desarrollo de un sensor de monóxido de carbono químico-resistivo. Estos permiten el calentamiento de un área de $1 \mathrm{~mm} \times 1 \mathrm{~mm}$ hasta una temperatura de $490^{\circ} \mathrm{C}$ sin apenas disipación de calor al área circundante.
\end{abstract}

Palabras clave: Cerámicas LTCC, micromecanizado láser, sensores de gas.

Femtosecond laser micromachining of LTCC ceramics. Fabrication of microhotplates for a carbon monoxide sensor

In present work, the processability of LTCC (Low Temperature Co-fired Ceramics) by means of femtosecond laser ablation is shown. This kind of material, with a clear technological interest in the development of microsensors, possesses a high mechanical fragility in the sintered state and, consequently, contact methods for micromaching (micromilling, microgrinding, etc.) lead to fracture or the appearance of microcracks. The paper has been structured in two different parts. Firstly, a detailed study of the process has been performed, obtaining the fundamental parameters, as ablation threshold and incubation factor and a parametrization of width and height of microchannels as a function of fluence and feedrate. Afterwards, these results have allowed the fabrication of simple structures as boxes, passing-through holes and $2^{1} /{ }_{2} \mathrm{D}$ structures. Finally, the technological interest of the material and the process is shown. This way, microheaters in LTCC have been fabricated as initial stage of a carbon monoxide chemoresistive sensor. The system allows the heating of a $1 \mathrm{~mm} \times 1 \mathrm{~mm}$ area up to $490^{\circ} \mathrm{C}$ with almost no heat dissipation to the surrounding area.

Keywords: LTCC ceramics, laser micromachining, gas sensors.

\section{INTRODUCCIÓN}

Las cerámicas de sinterizado a baja temperatura (Low Temperature Cofired Ceramics, LTCC) han despertado gran interés en los últimos años tanto en el mundo de la microelectrónica como en el de las microtecnologías. Esto es debido principalmente a (i) sus bajas temperaturas de sinterizado (menores de $900^{\circ} \mathrm{C}$ ), lo que hace que, con anterioridad a este proceso, puedan ser impresas mediante deposición de capas metálicas, (ii) la posibilidad de empaquetar varias de estas capas previamente al sinterizado, lo que da lugar a estructuras multicapa y (iii) la facilidad del material una vez sinterizado para ser pegado anódicamente al silicio (1). De acuerdo a estas propiedades, es posible la miniaturización de sistemas electromecánicos como acelerómetros, sensores, giróscopos, etc. hasta lo que se conocen como MEMS (Microelectromechanical Systems). (2, 3). Desde el punto de vista estructural, este tipo de cerámicas están basadas en una composición de alúmina, que le da su carácter cerámico, y una combinación de vidrios que reducen su temperatura de sinterizado, disminuyen su coeficiente de expansión térmica y aportan iones $\mathrm{Na}^{+}$para su procesabilidad mediante pegado anódico.

Además de las propiedades exhibidas por las cerámicas LTCC para su procesado en el estado verde, el material conserva interesantes características una vez sinterizado, 
por lo que, aunque menos, ha sido también utilizado para la fabricación de sensores. En este caso, el material es usado a modo de substrato como alternativa al silicio. Sin embargo, en este estado, el procesado del material es mucho más problemático debido principalmente a su alta fragilidad, haciéndose este hecho más patente cuando se trabaja con obleas de pocas centenas de micras de espesor. En esta situación, para cualquier microtecnología basada en la máquina-herramienta (microfresado, microtorneado, etc.) los esfuerzos de corte provocan la fractura de la oblea o, en las condiciones más favorables, la aparición de grietas y desconchamientos. Si se consideran otro tipo de tecnologías, como los ataques químicos o por plasma, no existe actualmente un conocimiento claro acerca de los reactivos líquidos o gaseosos que deben utilizarse.

El presente trabajo propone como aspecto más innovador el uso de la tecnología láser para la realización de microestructuras en cerámicas LTCC sinterizadas. En particular, se analiza el comportamiento de este material frente a pulsos láser ultracortos (femtosegundos). Los láseres de femtosegundos permiten eliminar los efectos térmicos comúnmente observados en el mecanizado láser convencional, por lo que han despertado una gran atención en los últimos años, especialmente en el mundo de las micro y nanotecnologías. En la actualidad estos sistemas vienen siendo utilizados para la fabricación de microsistemas electromecánicos, fotónicos o biomédicos (ver por ejemplo, $(4,5)$ ), así como en el procesado de materiales (6). La corta duración de estos pulsos $\left(<10^{13} \mathrm{~s}\right)$ hace evitar la transferencia de energía a la red cristalina y, aunque las bases microscópicas del proceso son objeto de debate en la actualidad, parece bien establecido que los mecanismos de absorción de la radiación en este caso difieren sustancialmente de los implicados cuando pulsos de mayor longitud temporal son utilizados. De este modo, las altas potencias de pico de estos láseres $(>1 G W)$ y la subsecuente extremadamente alta intensidad $\left(>1 \mathrm{TW} / \mathrm{cm}^{2}\right)$ conducen a procesos de ionización (multifotónica o por avalancha) en lugar de provocar cambio de fase termodinámicos. Por otro lado, la utilización de pulsos de corta longitud de onda (ultravioleta, visible) permite la minimización del tamaño del spot hasta tamaños cercanos a la micra.

Además de un estudio exhaustivo del proceso de ablación láser, el presente trabajo muestra una aplicación práctica, como es la fabricación de microcalentadores en membranas suspendidas para su utilización en sensores de monóxido de carbono. De manera resumida, puede decirse que dentro de los microsensores de gas, los de tipo químico-resistivo poseen una especial importancia debido a su simplicidad. En estos, la reacción química de adsorción entre una capa sensora de un determinado material y el gas provoca una variación en las propiedades eléctricas de la capa que puede ser detectada fácilmente mediante la colocación de dos electrodos en la misma. Dado que para que este tipo de reacciones se produzcan es necesario que la capa sensora se encuentre a temperaturas mayores de $400^{\circ} \mathrm{C}$, en todos los casos se hace necesario el diseño previo de un sistema de microcalentadores capaces de proporcionar estas temperaturas en superficies de pocos $\mathrm{mm}^{2}$ y con ausencia de transmisión de calor al resto del sistema $(7,8)$. Tradicionalmente, el sustrato elegido para la fabricación de estos dispositivos ha sido el silicio, si bien en los últimos años han venido introduciéndose sustratos cerámicos debido a sus buenas propiedades térmicas. En el presente trabajo, se muestra la fabricación mediante mecanizado láser de un microcalentador realizado sobre LTCC de $2 \mathrm{~mm} \times 2 \mathrm{mms}$ y suspendido en una membrana. Actualmente, este sistema está siendo integrado en la fabricación de un microsensor de monóxido de carbono con óxido de estaño $\left(\mathrm{SnO}_{2}\right)$ como capa sensora.

\section{EXPERIMENTAL}

Para la realización de las pruebas de micromecanizado sobre LTCC se ha utilizado un sistema láser comercial de Titanio:zafiro compuesto por tres etapas. Como primera de ellas, un oscilador (Coherent Vitesse LP SB) proporciona una potencia media de salida de $250 \mathrm{~mW}$ a una frecuencia de pulsado de $80 \mathrm{MHz}$ y con una anchura de pulso de 90fs. Con el fin de obtener potencias aceptables para el micromecanizado, este haz es introducido en un amplificador multipaso (Quantronix, Odin-Compact DP 1.0) basado en tecnología CPA (Chirped Pulse Amplification). De este modo, la energía de pulso aumenta desde $4 \mathrm{~nJ}$ hasta $1 \mathrm{~mJ}$, con una frecuencia de pulsado de $1 \mathrm{KHz}$ sin cambiar apreciablemente la forma y anchura del pulso. Por último, la frecuencia natural del cristal de titanio:zafiro es doblada mediante técnicas de generación de segundo armónico. Desde este modo, el haz resultante para el micromecanizado es gaussiano $\left(\mathrm{TEM}_{00^{\prime}}\right.$ $\mathrm{M}^{2}<1.3$ ), circular ( $3 \mathrm{~mm}$ de diámetro), con longitud de onda de 400 nm, energía máxima de pico de $350 \mu \mathrm{J}$ y frecuencia de pulsado de $1 \mathrm{KHz}$. La cantidad de energía en la muestra es controlada mediante la rotación de una lámina $\lambda / 2$ colocada antes del cristal LBO que provoca la variación del ángulo de polarización del haz $y$, consecuentemente, la variación de la eficiencia en la conversión de energía. De este modo, es posible el control de la energía desde $0 \mu \mathrm{J}$ hasta $350 \mu \mathrm{J}$ en pasos de $1 \mu \mathrm{J}$. Finalmente, se utiliza una lente de sílice fundida para el enfoque del haz sobre la muestra, trabajándose siempre en enfoque directo (sin técnicas de proyección de imagen). Para una descripción más detallada del sistema véase (9).

La cerámica LTCC utilizada en nuestros experimentos ha sido adquirida a Via Electronic $\mathrm{GmbH}$ (Alemania). Su composición está basada en alúmina, borosilicatos, cordierita y vidrios de bajo coeficiente de expansión térmica que igualan el de el conjunto al del silicio a fin de poder ser utilizado este material en procesos de pegado anódico. El tamaño de grano del conjunto con anterioridad al sinterizado se ha estimado en $600 \mathrm{~nm}-700 \mathrm{~nm}$. Los porcentajes exactos de cada compuesto no han sido proporcionados por el fabricante.

\section{RESULTADOS}

Los resultados obtenidos se presentan en dos bloques bien diferenciados. En el primero, se muestran los estudios iniciales realizados en los que, además de extraer los parámetros fundamentales del proceso (umbral de ablación, coeficiente de incubación, etc.), se analizan la estrategia de micromecanizado y se muestran algunas estructuras elementales (cajeras, orificios y estructuras $2^{1 /}$ D) fabricadas. En la segunda parte, se presentan los microcalentadores para un sensor de gas fabricados mediante la conjunción de la ablación láser y la técnica de screen-printing. 


\subsection{Estudio del proceso de mecanizado en LTCC}

\subsubsection{Parametrización del micromecanizado de canales} en LTCC

Primeramente, se han obtenidos los parámetros fundamentales del proceso de ablación, gracias a la realización de una matriz de cráteres producidos mediante la focalización de un número de pulsos, $\mathrm{N}$, con una determinada densidad de energía, F. Así, se han realizado cráteres para $\mathrm{N}=1,20,100$ y 1000 pulsos y $\mathrm{F}=2.6 \mathrm{~J} / \mathrm{cm}^{2}, 4.2 \mathrm{~J} / \mathrm{cm}^{2}, 7.6 \mathrm{~J} / \mathrm{cm}^{2}$ y $15.8 \mathrm{~J} / \mathrm{cm}^{2}$. Analizando los diámetros de estos cráteres y ajustando a las leyes que gobiernan el comportamiento de este parámetro en función de la densidad de energía para un haz gausiano, se ha obtenido el diámetro del spot focalizado sobre la superficie del material. Este es de $32.1 \mu \mathrm{m} \pm 0.5 \mu \mathrm{m}$ y está en acuerdo con el valor esperado de acuerdo a la teoría de la difracción para un haz con las características señalas en la sección experimental. Del mismo modo, se ha obtenido el umbral de ablación (menor densidad de energía para la que el fenómeno de ablación tiene lugar), $F_{t h}$. Este valor generalmente depende del número de pulsos, $\mathrm{N}$, por lo que en general se define un umbral de ablación, $F_{t h}(N)$ que viene dado por una ley de potencias $\mathrm{F}_{\mathrm{th}}(\mathrm{N})=\mathrm{F}_{\mathrm{th}}(1) \mathrm{N}^{\xi-1}$, siendo $\mathrm{F}_{\mathrm{th}}(1)$ y $\xi$ valores característicos del material. En el caso del LTCC, se ha obtenido $F_{t h}(1)=1.59 \mathrm{~J} / \mathrm{cm}^{2}$ y $\xi=0.87$. Para un estudio detallado del proceso de análisis y extracción de estos parámetros, véase (10).

Con el fin de caracterizar el proceso de micromecanizado, se han realizado canales para diferentes densidades de energía y velocidades de avance de la muestra respecto del haz, f. Aunque la dependencia funcional de la anchura y la altura de los canales, $\omega(\mathrm{f}, \mathrm{F})$ y $\mathrm{h}(\mathrm{f}, \mathrm{F})$ respectivamente, viene dada por expresiones complicadas (10), en el presente trabajo se ha llevado a cabo un proceso de parametrización de estos valores a ecuaciones sencillas, válidas en los intervalos $0.10 \mathrm{~mm} /$ $\mathrm{s}<\mathrm{f}<1.25 \mathrm{~mm} / \mathrm{s}$ y $6 \mathrm{~J} / \mathrm{cm}^{2}<\mathrm{F}<32 \mathrm{~J} / \mathrm{cm}^{2}$. Así, la anchura y altura de los canales fabricados han sido ajustadas a expresiones en las que se ha considerado hasta el término cuadrático tanto de $\mathrm{F}$ como de $\mathrm{f}$, así como los términos cruzados. El ajuste ha sido realizado mediante mínimos cuadrados en el que todos los puntos experimentales han sido considerados con igual peso. En la Figura 1(a) se representan las anchuras de los canales realizados para diferentes valores de $\mathrm{f}$ y $\mathrm{F}$. Dada la leve dependencia de este parámetro observada como función del avance, se ha decidido centrar el estudio en valores altos de $\mathrm{f}$, dado que de este modo se minimiza el tiempo de mecanizado de manera significativa. En la Figura 1(b) se muestran los valores de las alturas de los canales en función de la densidad de energía para diferentes valores del avance mayores de $\mathrm{f}=0.5 \mathrm{~mm} / \mathrm{s}$. Como se ha comentado anteriormente, ambos parámetros han sido modelizados por expresiones sencillas que predicen su comportamiento con una resolución aproximadamente igual al error experimental.Estas, representadas por líneas sólidas en las figuras anteriormente mencionadas, pueden escribirse como:

$\omega(f, F)(\mu m)=16.84+2.18 F\left(J / \mathrm{cm}^{2}\right)-3.95 f(\mathrm{~mm} / \mathrm{s})$

$$
\begin{aligned}
& h(f, F)(\mu \mathrm{m})=\left(30.83-38.72 f(\mathrm{~mm} / \mathrm{s})+14.40 f(\mathrm{~mm} / \mathrm{s})^{2}\right)+ \\
& +\left(1.86-2.32 f(\mathrm{~mm} / \mathrm{s})+0.90 f(\mathrm{~mm} / \mathrm{s})^{2}\right) F\left(\mathrm{~J} / \mathrm{cm}^{2}\right)
\end{aligned}
$$
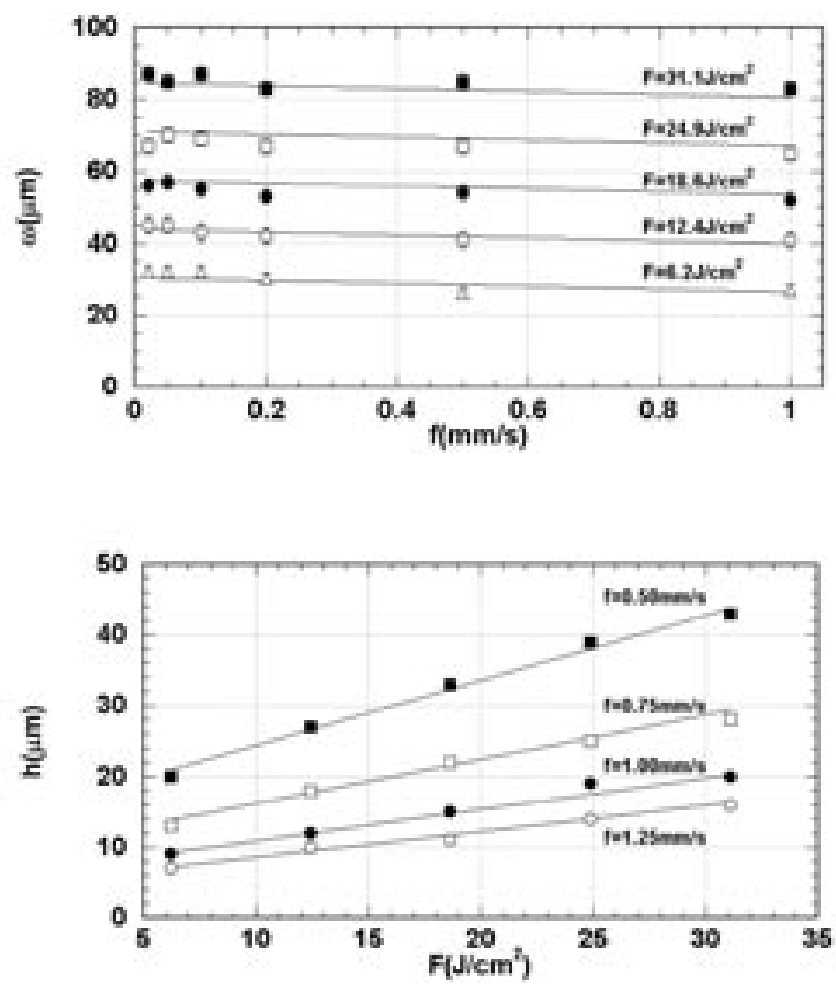

Fig. 1- Anchura (a) y altura (b) de los canales micromecanizados para diferentes valores de la densidad de energía y del avance. Las líneas continuas se corresponden con las expresiones [1] y [2] del texto.

Los coeficientes de regresión obtenidos han sido en ambos casos mejores que 0.99 . Los términos no significativos han sido suprimidos en estas expresiones para mayor claridad.

\subsubsection{Fabricación de orificios, cajeras, estructuras $2^{1 / 2} \mathrm{D}$}

Una vez caracterizado el comportamiento de la cerámica LTCC frente a la ablación láser con pulsos ultracortos, se ha procedido al mecanizado de algunas estructuras sencillas como las que se muestran en la Figura 2. Estas imágenes han sido obtenidas mediante una cámara CCD de alta definición con una óptica 0.75X-4.5X. En la Figura 2(a) se muestra una cajera de $1 \mathrm{~mm} \times 1 \mathrm{~mm}$ realizada de acuerdo a una estrategia de mecanizado consistente en la superposición de canales separados la sexta parte de su anchura. La densidad de energía ha sido de $24.9 \mathrm{~J} / \mathrm{cm}^{2}$ y el avance de $1 \mathrm{~mm} / \mathrm{s}$. La irradiación del material en estas condiciones da lugar a espesores de $57 \mu \mathrm{m}$, por lo que la altura final conseguida $(302 \mu \mathrm{m})$ se alcanzó mediante 5 repeticiones del proceso (proceso capa a capa). La Figura 2(b) se muestran orificios pasantes de $500 \mu \mathrm{m}$ de diámetro obtenidos con valores de $\mathrm{F}$ y f similares al caso anterior y micromecanizados mediante trepanado circular. El orificio de salida posee un diámetro de $410 \mu \mathrm{m}$ por lo que, teniendo en cuenta que el espesor del material es de $560 \mu \mathrm{m}$, el ángulo de la pared es de $85^{\circ}$, aproximadamente. Igualmente, se ha realizado una estructura en " $\mathrm{V}$ " mediante el mecanizado capa a capa, tal y como se muestra en la Figura 2(c). Las dimensiones de la estructura son de $2 \mathrm{~mm} \times 1 \mathrm{~mm}$ con una altura en el centro de $200 \mu \mathrm{m}$ que disminuye linealmente hasta hacerse cero en los extremos. 
Con el fin de mostrar la calidad del mecanizado obtenido en este tipo de material mediante pulsos ultracortos, la Figura 3 muestra un detalle de los orificios de entrada y de salida en la Figura 2(b). Las imágenes han sido obtenidas mediante microscopía confocal y, como puede apreciarse, en ninguno de
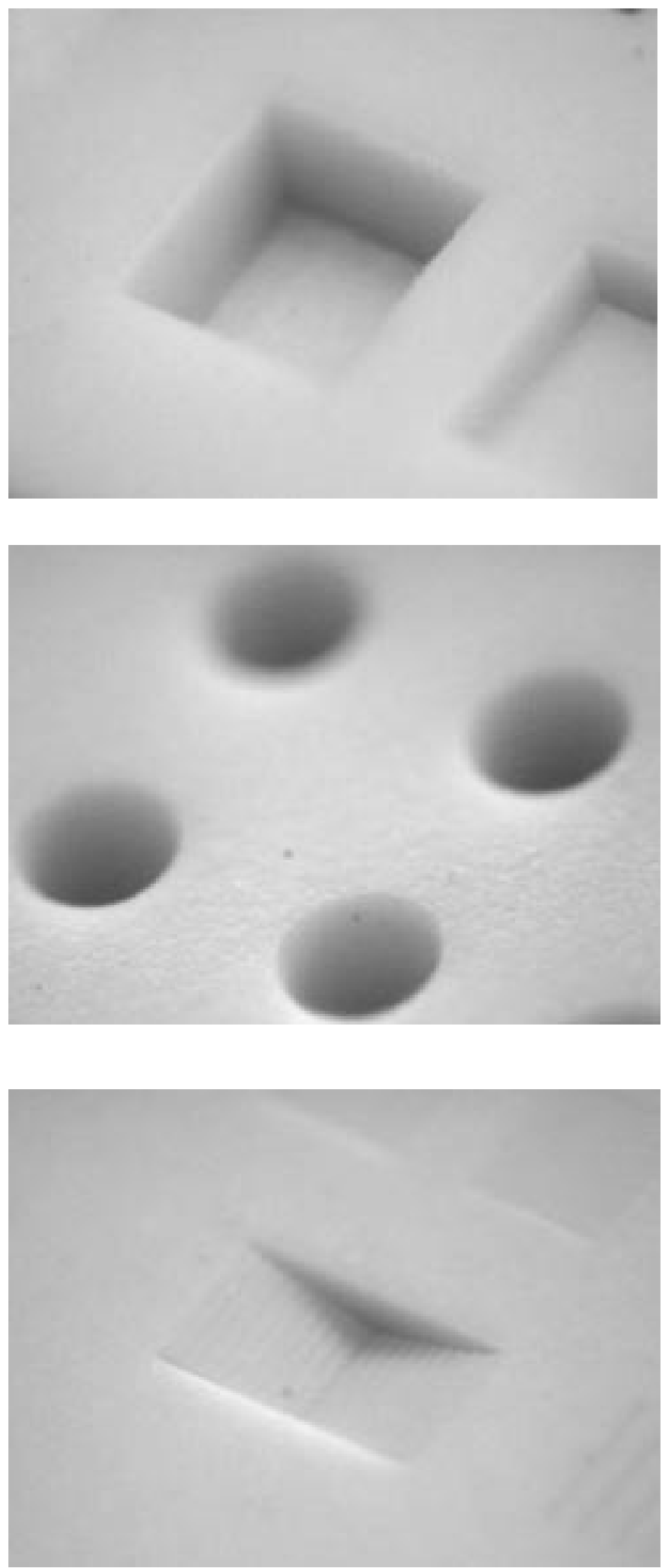

Fig. 2- Algunos ejemplos de mecanizado: (a) cajeras de $1 \mathrm{~mm} \times 1 \mathrm{~mm}$ x $0.3 \mathrm{~mm}$, (b) orificios pasantes de $500 \mu \mathrm{m}$ de diámetro y (c) estructura en "V".
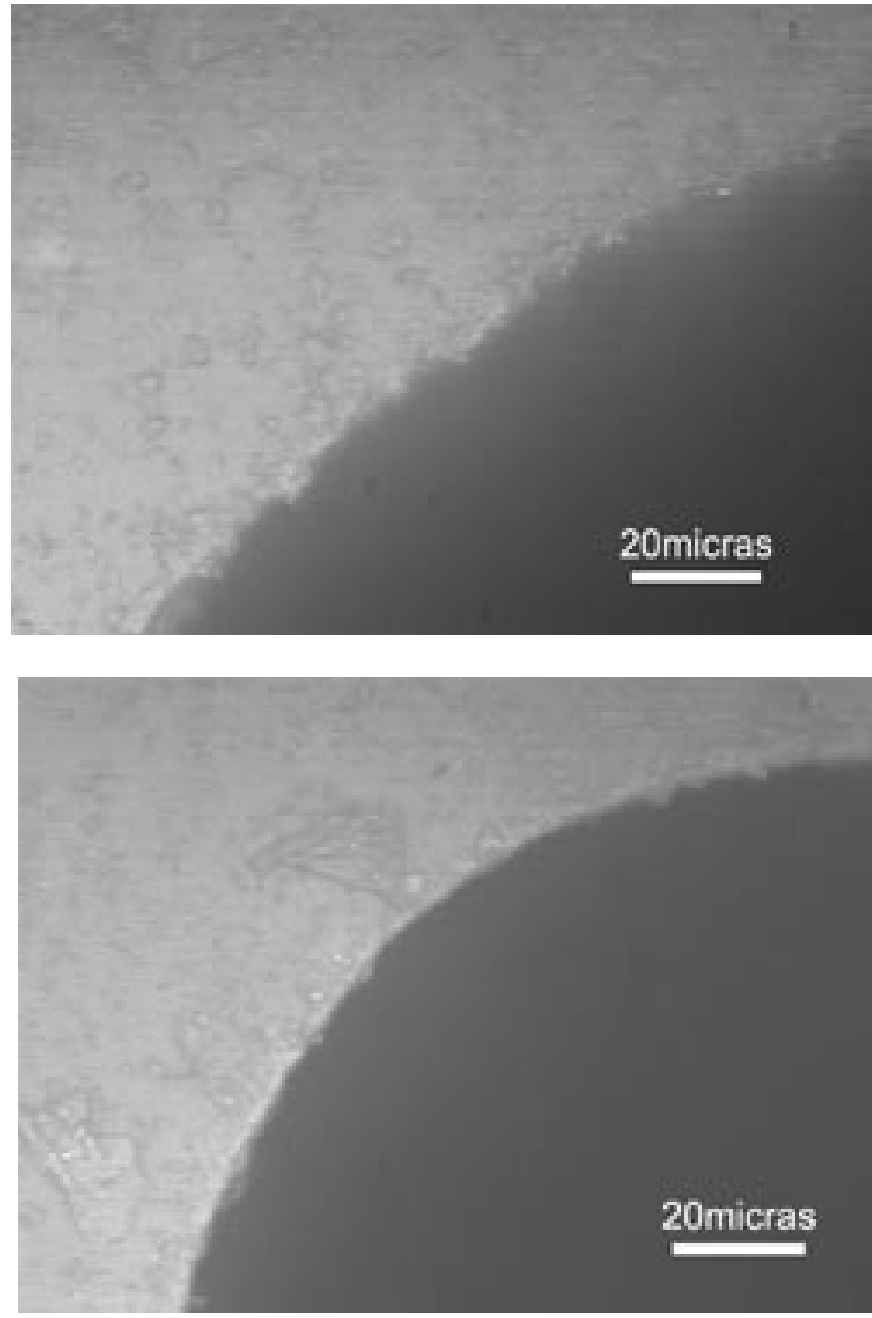

Fig. 3- Detalle de los orificios de entrada (a) y salida (b) correspondientes a la Figura 2(b) obtenidos mediante microscopía confocal.

los casos existen zonas afectadas por el calor, microfracturas o grietas, típicas en el procesado mediante láseres de otras características.

\subsection{Microcalentadores para sensores de monóxido de carbono}

Como último punto de este apartado, se muestra una aplicación práctica en la que se pone de manifiesto la utilidad de las cerámicas LTCC en el desarrollo de microsensores. Como ya ha sido comentado en la introducción, los sensores de gas químico-resistivos precisan de la fabricación previa de microcalentadores que permitan el aumento de la temperatura de la capa sensora hasta valores en los que se produce la adsorsión del gas a detectar (típicamente, $400^{\circ} \mathrm{C}-600^{\circ} \mathrm{C}$ ). En el presente trabajo se ha diseñado y fabricado un microsistema calefactor que, de manera básica, puede definirse como una resistencia eléctrica suspendida en una membrana con el fin de minimizar la disipación de calor. En la Figura 4 se muestran de manera esquemática las etapas de fabricación. Así, se parte de un sustrato de LTCC (Figura 4(a)), que es micromecanizado mediante ablación láser con una estructura en forma de serpentín de $1 \mathrm{~mm}$ x $1 \mathrm{~mm}$ y dos extremos más anchos que actúan como contacto con los electrodos 


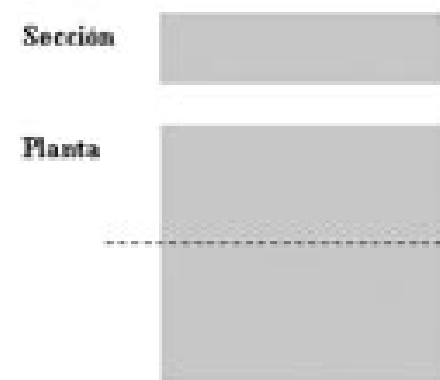

(a)
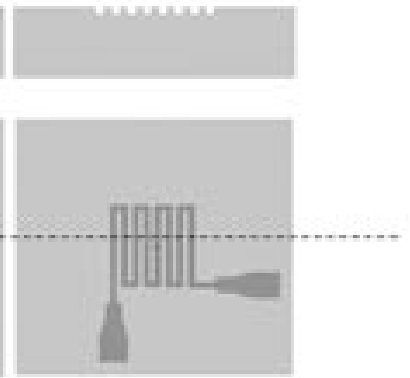

(b)

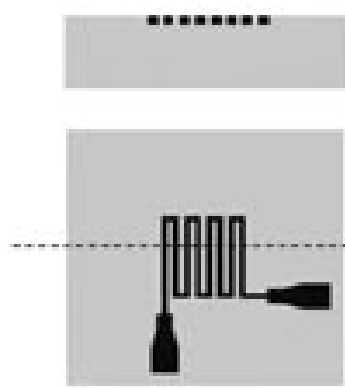

(c)
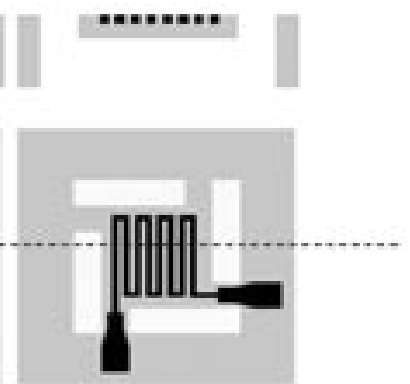

(d)

Fig. 4- Secuencia de fabricación de un microcalentador en sustrato de LTCC. Se muestra la planta y la sección del sustrato a lo largo de la línea de puntos señalada.

eléctricos (Figura 4(b)). Dado que los cálculos realizados precisan canales en torno a $90 \mu \mathrm{m}$ de anchura y $40 \mu \mathrm{m}$ de profundidad, de acuerdo a los resultados obtenidos en el apartado anterior, se ha elegido una densidad de energía de $32 \mathrm{~J} / \mathrm{cm}^{2}$ con un avance de $0.5 \mathrm{~mm} / \mathrm{s}$ para su realización. Posteriormente, los canales realizados son rellenados por una pasta conductora de oro, platino y paladio mediante la tecnología de screen-printing (Figura 4(c)). Finalmente, la resistencia eléctrica creada es aislada térmicamente del resto del sustrato mediante la fabricación de una membrana (Figura 4(d)). Las imágenes mostradas en la Figura 5, obtenidas de modo análogo a las mostradas en la Figura 2, muestran la cara superior e inferior del dispositivo fabricado. Cabe señalar que se ha realizado una caracterización del sistema mediante una cámara térmica, obteniéndose temperaturas estables en la membrana en torno a $490^{\circ} \mathrm{C}$ para una potencia eléctrica aplicada de 1.1W (Figura 6) y con escasa disipación de calor al área circundante. Experimentos similares realizados sobre resistencias sin aislar mediante una membrana muestran que en este caso son necesarios 24W. Actualmente, se está procediendo a la fabricación final del sensor a partir de estos microcalentadores.

\section{CONCLUSIONES}

En este trabajo, se muestra la importancia de los láseres de femtosegundos para el microprocesado de cerámicas LTCC en especial si se tienen en cuenta las propiedades mecánicas de este tipo de materiales. Como ha sido presentado, la
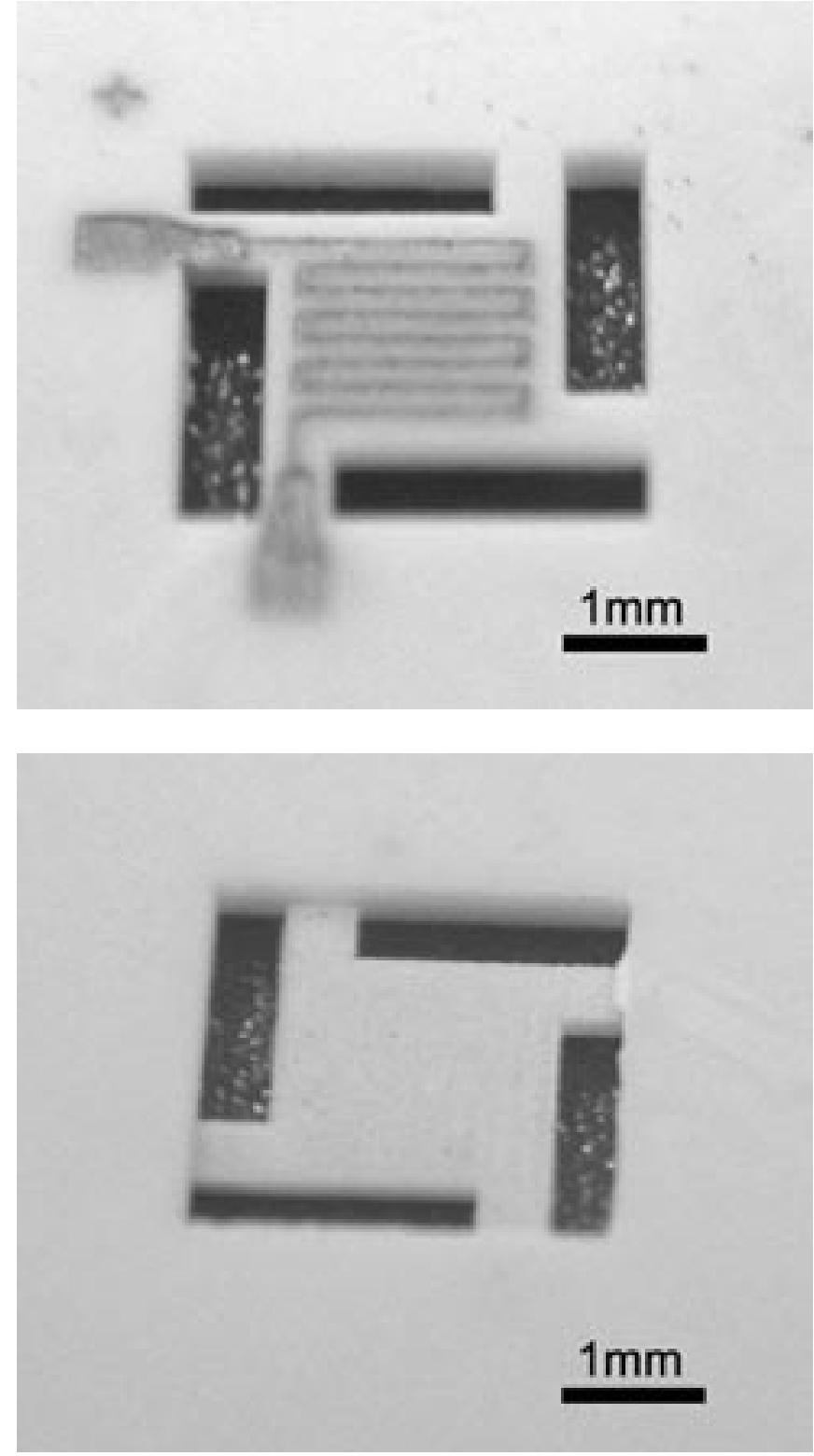

Fig. 5- Imágenes de la parte superior (a) e inferior (b) de los microcalentadores fabricados.

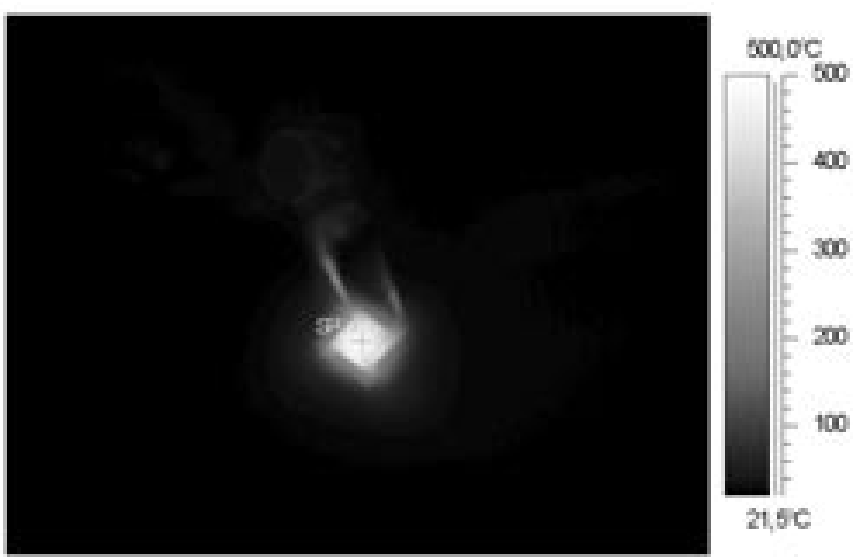

Fig. 6- Imagen obtenida mediante una cámara infrarroja en la que se muestra el comportamiento del microcalentador para una potencia de $1.1 \mathrm{~W}$. 
ablación láser con pulsos ultracortos conduce a resultados satisfactorios en cuanto a la minimización de zonas afectadas por el calor, ausencia de grietas o reducción de las dimensiones del mecanizado hasta valores de pocas decenas de micras. Gracias a estas características, ha sido posible la obtención de membranas de $200 \mu \mathrm{m}$ de espesor, $1 \mathrm{~mm}^{2}$ de sección y suspendidas en brazos de $200 \mu \mathrm{m}$ de anchura sobre las que han sido estructuradas resistencias eléctricas, dando lugar a lo que se conoce en el mundo de la detección de gases como microcalentadores. En las actualidad, estos dispositivos están siendo integrados en sensores de monóxido de carbono.

\section{BIBLIOGRAFÍA}

1. E. Müller, T. Bartnitzek, F. Bechtold, B. Pawlowski, P. Rothe, R. Ehrt, A. Heymel, E. Weiland, T. Schroeter, S. Schundau, K. Kaschlik. "Development and processing of an anodic bondable LTCC tape". Proceedings of the 15th European Microelectronics and Packaging Conference 2005, Brujas, Bélgica.

2. M. Góngora-Rubio, L.M. Solá-Laguna, P.J. Moffet, J.J. Santiago-Avilés. "The utilization of low temperature co-fired ceramics (LTCC-ML) technology for meso-scale EMS, a simple thermistor based flow sensor" Sensors and Actuators 73, 215-221 (1999)
3. M. Góngora-Rubio, P. Espinoza-Vallejos, L.M. Solá-Laguna, J.J. Santiago-Avilés. "Overview of low temperature co-fired ceramics tape technology for meso-system technology (MsST)" Sensors and Actuators A 89, 222-241 (2001)

4. R. Fedosejevs, M. Argument, A. Sardarli, S.E. Kirkwood, R. Holenstein, Y.Y. Tsui. "Laser micromachining for microfluidic, microelectronic and MEMS applications". Proceedings of the International Conference on MEMS, NANO and Smart Systems, 2003, p. 53.

5. P.R. Herman, K.P. Chen, P. Corkum, A. Naumov, S. Ngi, J. Zhang "Advanced lasers for photonic device microfabrication". RIKEN Rev. 32 (2001) 31-35.

6. P. Moreno, C. Méndez, A. García. “Femtosecond laser micromachining of ceramics". Bol. Soc. Esp. Ceram. V., 44(1), 9-12 (2005)

7. L. Zhu, C. Kimball, N. Suiadecki, M. Beamesderfer, S. Semancik, D.L. DeVoe. "Integrated microfluidic gas sensors for water monitoring". Proceedings of the 7th Internacional Conference on Miniaturizad and Biochemical Análisis Systems, Squaw Valley, California (USA) (2003)

8. D. Vicenzi, M.a. Butturi, V. Guidi, M.C. Carotta, G. Martinelli, V. Guarnieri, S. Brida, B. Margesin, F. Giacomozzi, M. Zen, D. Giusti, G. Soncini, A. A. Vasiliev, A. V. Pisliakov. "Gas-sensing device implemented on a micromachined membrane: A combination of thickfilm and very large scale integrated technologies". Journal of Vacuum Science \& Technology B: Microelectronics and Nanometer Structures, 18 (5) 2441-2445 (2000)

9. D. Gómez, I. Goenaga, I. Lizuain, M. Ozaita. "Femtosecond Laser Ablation for microfluidics". Optical Engineering 44(5), 051105(2005)

10. D. Gómez, I. Goenaga. "On the incubation effect on two thermoplastics when irradiated with ultrashort laser pulses: broadening effects when machining microchannels". Applied Surface Science 253, 2230 (2006) 